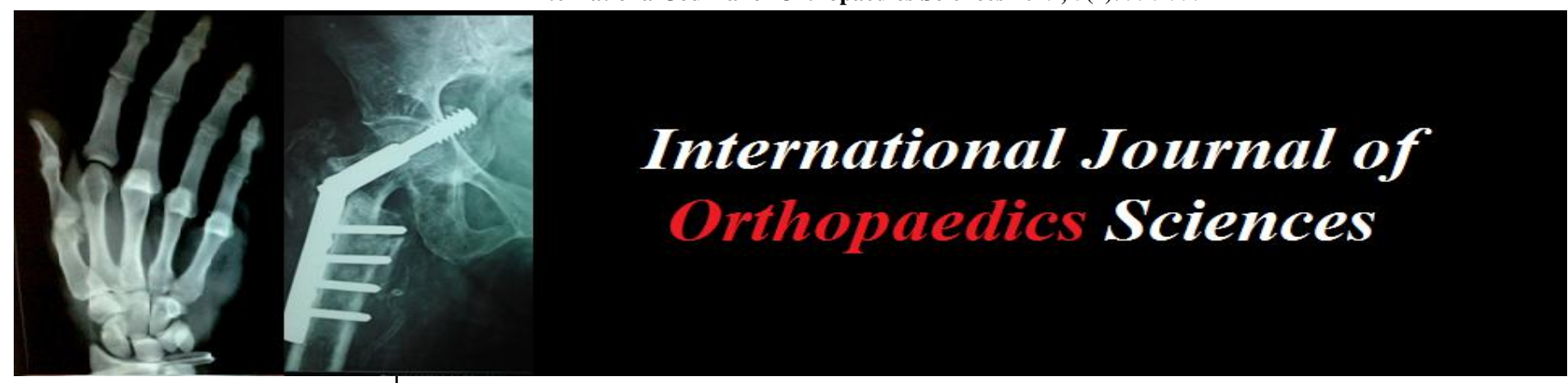

E-ISSN: 2395-1958

P-ISSN: 2706-6630

IJOS 2019; 5(4): 995-999

(C) 2019 IJOS

www.orthopaper.com

Received: 20-08-2019

Accepted: 22-09-2019

Dr. Chandrasen I Chaughule Orthopaedic Resident,

Department of Orthopaedics,

Maharashtra University of

Medical Sciences, Maharashtra, India

Dr. Nitin A Bhalerao

M.S. Ortho, Department of Orthopaedics, Maharashtra University of Medical Sciences,

Maharashtra, India
Corresponding Author: Dr. Chandrasen I Chaughule Orthopaedic Resident, Department of Orthopaedics, Maharashtra University of Medical Sciences, Maharashtra, India

\section{Meralgia paraesthetica following lumbar spine surgery: A study in 120 consecutive surgically treated cases}

\author{
Dr. Chandrasen I Chaughule and Dr. Nitin A Bhalerao
}

DOI: https://doi.org/10.22271/ortho.2019.v5.i4q.1808

\section{Abstract}

Background: Meralgia paresthetica is a non-life-threatening neurological disorder characterized by numbness, tingling, and burning pain over the anterolateral aspect of thigh due to impingement of the lateral femoral cutaneous nerve. This disorder has been seen in patients with diabetes mellitus and obesity, but has also been observed in patients after procedures such as posterior spine surgery, iliac crest bone grafts, lumbar disk surgery, hernia repair, appendectomies, and pelvic osteotomies that ultimately lead to compression or damage to the lateral femoral cutaneous nerve. Overall, permanent sequelae of meralgia paresthetica are rare, however, some cases do require intervention.

Study design: In this prospective study of 120 patients who underwent elective lumbar spinal surgery, the incidence, possible risk factors, etiopathogenesis and management of MP were analysed.

Result: There were 70 males and 50 females. The age of the patients ranged from 15 to 81 years (mean 46.9 yrs.). Thirteen patients (12\%) suffered from MP. It is more common in thinner individuals due to pressure injury to the nerve at its exit point. Ninety-two percent of the patients were asymptomatic at follow-up after 6 months. In 7 out of 13 patients, patchy sensory loss on clinical examination was seen at 6 months.

Conclusion: MP after posterior lumbar spinal surgery is uncommon. Smaller bolsters may avoid some of the vulnerable pressure points, as the surface area available is relatively smaller. The posts of the HallRelton frame over the anterior superior iliac crest should be adequately padded. The condition is usually self-limiting. Surgical division or decompression of the LCFN is reserved for persistent or severe MP.

Keywords: Meralgia paraesthetica, lateral femoral nerve, prone position, spinal surgery

\section{Introduction}

Meralgia paraesthetica (MP) also known as Bernhardt Roth Syndrome ${ }^{[1]}$ is a clinical syndrome resulting from the entrapment of the lateral femoral cutaneous nerve (LFCN) of the thigh in the inguinal region ${ }^{[2-4]}$. The term "meralgia paraesthetica" combines four Greek roots to mean "thigh pain with anomalous perception". The disorder has also been nicknamed Skinny Pants Syndrome ${ }^{[5]}$, in reference to a rise in teenagers wearing skin-tight trousers. It is characterized as being disagreeably numb or a burning, tingling or pricking sensation over the anterolateral aspect of one or rarely both thighs. It is commonly observed following chronic irritation of the nerve due to mechanical factors exerting a downward pull or traction on the inguinal ligament. Obesity, pulmonary obstructive disease with a chronic cough and anatomical variations in the course of the nerve are common predisposing factors. The occurrence of MP following lumbar spine surgery is uncommon and there are few reports in the literature on this subject ${ }^{[6]}$.

Pathophysiology and anatomy: Originating from the second and third lumbar roots, the LFCN emerges from the lateral side of the psoas major muscle and crosses the ilium to pass under the lateral end of the inguinal ligament at the site of its attachment to the anterior superior iliac spine (ASIS). The nerve is located in the angle between the ligament, the bone, and the sartorius muscle. Proceeding distally, the LFCN enters the thigh beneath the fascia lata and provides sensory innervation to the lateral and anterolateral regions of the thigh. The course of the LFCN may exhibit 4 possible variations in relation to the ASIS and its exit point. Nerve A crosses over the iliac crest $>2 \mathrm{~cm}$ posterior to the ASIS. Nerve B crosses over the iliac crest $<2 \mathrm{~cm}$ posterior to the ASIS. Nerve $C$ crosses at the ASIS. Nerve D crosses under the inguinal ligament and anterior to the ASIS. As the LFCN is most vulnerable to compression 
and entrapment at its exit point, the variability of the nerve's course may be a contributing factor of prone position-related MP in patients who have had posterior spine surgery as well as by the presence of a neurinoma formation. Williams and Trzil have described the following anatomic variations of the LFCN: "the LFCN passing through a notch between the ASIS and anterior inferior iliac spine; the LFCN exiting the pelvis over the iliac crest with neurinoma formation; the LFCN exiting the pelvis through the inguinal ligament with neurinoma formation; the LFCN exiting the pelvis as 2 branches with neurinoma formation on 1 branch; the LFCN exiting the pelvis as 3 branches with neurinoma formation on the middle branch; the LFCN exiting the pelvis far medially in close proximity to the femoral nerve".

\section{Risk factor}

- Patients Body Weight : Thin/Obese

- Spinal Abnormalities like idiopathic scoliosis, congenital scoliosis, neuromuscular scoliosis or kyphoscoliosis

- Age

- Diabetes/Hypertension/Other Neuropathy

- Type/Duration of surgery

Risk reduction: To minimize the occurrence of prone position-related MP, physicians should take note of certain preventative measures. The first of the preventative measures involves readjustment and proper positioning of the patient on the operating table. One review of 56 paediatric patients undergoing posterior spine surgery found that there was an increase in the number of patients diagnosed with MP postoperatively based on the configuration of the Hall-Relton frame typically used during these type of procedures. The 2 types of configurations used involved a flat table extending to the lower legs with pads on the thighs and the second configuration was a sling to support the lower legs. The patients positioned with the sling were found to have significantly higher incidence of MP compared with patients positioned on the flat table. This was thought to be due to the lack of thigh support pads in the sling configuration, which allowed for greater pressure on the ASIS thereby increasing the tension on the LFCN. Therefore, thigh pads should always be used in these types of surgeries to decrease the risk of MP. The patient should also be symmetrically positioned on the frame and there should be smaller bolsters supporting the ASIS along with sufficient distance between the pelvic posts. Intraoperative blood loss and length of surgery should be reduced as well.

Treatment: Conservative treatment is the first line approach to lessening symptoms of MP and includes tactics such as loosening tight clothing like low rise jeans or belts, applying ice to the anterolateral surface of the leg to decrease swelling, administration of anti-inflammatory medications and having the patient avoid physical exertion that requires any hip extension. Other conservative measures involved weight loss, topical application of lidocaine, or medication with Gabapentin, chlorpromazine, or antidepressants. If these conservative treatments do not resolve symptoms within 3 months, procedures such as a LFCN block using anaesthetics and steroids or pulsed radiofrequency neuromodulation treatments have been considered to avoid surgery and its subsequent risks. If these procedures do not work then surgical interventions need to be considered including neurolysis or a partial or complete resection of the LFCN especially if the symptoms become debilitating.
Since there were not many studies analysing the incidence, etiopathogenesis and management of this condition a prospective study is discussed and the relevant literature is briefly reviewed in the following section.

\section{Materials and Method}

One hundred and twenty patients of lumbosacral spine degenerative disease including high and low lumbar levels underwent surgery in the prone position over a period of six months. Preoperative neurological assessment was carried out with special attention to the sensory, motor system and the reflexes. The surgeries mainly included microlumbar discectomy, laminectomy, internal decompression for spinal stenosis, and posterior lumbar interbody fusion (Table 1). Microlumbar surgery was carried out through a small paramedian incision on one side of the spine. All the other procedures were carried out through midline incision. The operation time ranged from 90-500 minutes. Blood loss during surgery ranged from 100-400 ml. Clinical examination showed partial or dense sensory loss along the distribution of the lateral cutaneous nerve of the thigh on the anterolateral aspect of the thigh. Local anesthesia was not administered to produce relief of pain or paresthesias and confirm the diagnosis of MP. Neurophysiological examination including sensory nerve conduction or dermatome somatosensory evoked potentials was also not performed.

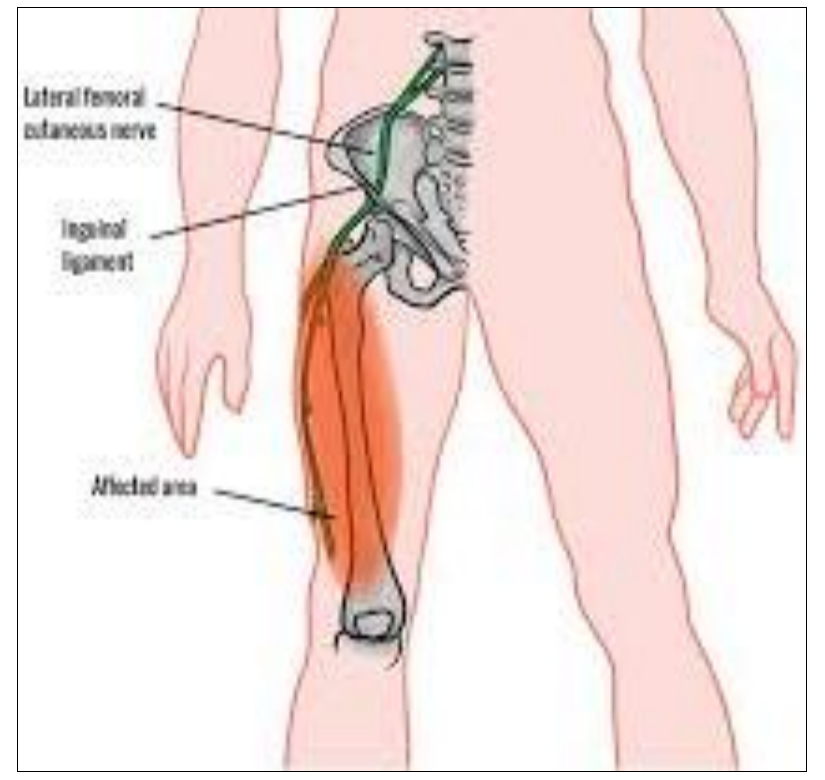

Fig 1: Meralgia paresthetica

Table 1: Type of lumbar surgeries

\begin{tabular}{|c|c|}
\hline Surgical procedures & $\begin{array}{c}\text { Number of } \\
\text { Patients }\end{array}$ \\
\hline Microlumbar discectomy & 53 \\
\hline Laminectomy & 32 \\
\hline IDSS: Internal decompression for spinal stenosis & 15 \\
\hline Instrumentation & 16 \\
\hline Others (tumors, dysraphism) & 4 \\
\hline
\end{tabular}

\section{Result}

There were 70 males and 50 females. The age of the patients ranged from 15 to 81 years (mean 46.9 yrs.). The weight of the patients ranged from 42 to $110 \mathrm{~kg}$ (mean $66.7 \mathrm{~kg}$ ). Nineteen patients (11 males and 8 females) suffered from diabetes mellitus and 32 patients suffered from hypertension (14 males and 18 females). Hypertension and diabetes mellitus were common in 14 patients (6 males and 8 females). 
All patients were assessed at follow-up after 1-6 months. The mean follow-up duration was 4 months. Thirteen patients complained of MP, 11 patients on one side and 2 patients on both sides. (Table 2) The patients mentioned about an unpleasant feeling in the same evening of the day of the operation or on the next day in the morning. The feelings were of two types: 9 patients complained of abnormal unpleasant paraesthesia and 4 patients complained of numbness over the anterolateral aspect of the thigh. (Table 3) Sixty-two percent (62\%) patients were relieved of MP in 10 days, $85 \%$ patients in 1 month and $92 \%$ patients were asymptomatic in 6 months duration. In the 8 patients who underwent microlumbar disc surgery, one had bilateral dysesthesia, 3 patients had symptoms on the same side as the disc prolapse and 4 patients on the opposite thigh. The ailment was seen more in younger patients than adults with an average age of 38.2 years as against the average age of 46.9 years in the series. Only 2 patients had hypertension and 1 patient had diabetes mellitus. It was more frequently seen in young thinner individuals (11 patients) than elderly obese patients ( 2 patients). The average weight of the thinner individuals developing MP was $45.2 \mathrm{~kg}$ in comparison to the average weight of $68.4 \mathrm{~kg}$ in those who did not develop MP. In 12 patients the abnormal feeling was seen within 12 hours and in 1 patient it was observed on the fourth day. The feeling was most intense on first day, became slightly less on the third day and on the tenth day $40 \%$ patients had totally improved and forgotten about the ailment. At 1 month, the abnormal feeling although present was not unpleasant in 3 patients and only 1 patient remembered the abnormal feeling at 6 months although sensory loss (patchy) in the distribution of the LCFN of the thigh was present in 7 patients. In 7 out of 13 patients, patchy sensory loss on clinical examination was seen at 6 months. Oral Gabapentin $300 \mathrm{mg}$ thrice a day for 3 weeks was administered empirically although no randomized trial has been conducted to study its efficacy in MP.

Table 2: Meralgia paraesthetica following lumbar surgery

\begin{tabular}{|c|c|}
\hline Surgical procedures & Number of patients \\
\hline Micro Lumbar discectomy & 8 (one bilateral) \\
\hline Laminectomy & 1 \\
\hline Instrumentation & 4 (one bilateral) \\
\hline
\end{tabular}

Table 3: Complete recovery after Meralgia paraesthetica

\begin{tabular}{|c|c|}
\hline Duration & Number of days \\
\hline 10 days & $8(62 \%)$ \\
\hline 30 days & $11(85 \%)$ \\
\hline 180 days & $12(92 \%)$ \\
\hline
\end{tabular}

\section{Discussion}

LCFN arises in the lumbar plexus from L2 and L3 nerve roots. It has a constant course in the iliac fossa. The nerve comes out piercing the Poupart's ligament medial to the anterior superior iliac spine remaining superficial to the sartorius muscle but deeper to the deep fascia. About 4 inches below this point it becomes subcutaneous. However, the exit point varies significantly. Aszmann et al. observed that in 5 of the 9 cadavers dissected, the exit of the nerve was more than 1 centimeter away from the anterior superior iliac spine. ${ }^{7}$ In another study of 52 human specimens the nerve in $54 \%$ of the cases was found to be directly susceptible to pressure ${ }^{[8]}$. De Ridder et al. found abnormal anatomy in $26 \%$ of cadavers ${ }^{[9]}$. The exit point of the nerve is most vulnerable and susceptible to compression and constitutes entrapment neuropathy ${ }^{[7]}$. Since MP is characterized by pure sensory symptoms, there is no muscle weakness produced by the entrapment of the nerve. It is frequently misdiagnosed and can be attributed to surgical sequelae. A rare occurrence observed by the senior author is the feeling of burning occurring several weeks after the surgery. It is diffuse and is not restricted to the distribution of the LFCN. Its late occurrence does not argue in favour of the entrapment of this nerve. An autonomic disturbance occurring following surgery is possible since it is relieved following a ganglion block. The common etiologies of LCFN entrapment include iliac crest bone graft harvesting and seat belt injury in a vehicular accident ${ }^{[10-12]}$. The exact significance of chronic medical disease, especially diabetes mellitus, and hypertension in the etiopathogenesis of MP is not clearly defined. The weight of the patient and the duration of the surgery may contribute proportionately towards the etiology or outcome of MP. In addition, it is highly probable that intraoperative hypotension and significant blood loss during surgery could make nerves more susceptible to compression. It is observed in both sexes and is more prevalent in thinner individuals suggesting the theory of direct compression of this delicate nerve in the prone position. The patient usually mentions about MP within hours of surgery or might observe it on the following morning after surgery. The symptoms can manifest even on the fifth day ${ }^{[13]}$. We preferred to administer Gabapentin in a small dose of $300 \mathrm{mg}$ three times a day for 3 weeks to these patients although its efficacy in MP is controversial. However, the results were encouraging in patients at the time of discharge from the hospital. They were quite happy, comfortable and required minimal reassurance. MP is an expression of pressure neuropathy caused by pressure of the bolster on the nerve at its exit and occurring in people sleeping prone on a wooden surface ${ }^{[14]}$. The pressure on the nerve causes injury to the nerve resulting in axonotmesis or neurotmesis. The final presentation of either numbness or unpleasant paraesthesia depends on the type of injury. It is reported to occur following frog leg position used in coronary bypass surgery ${ }^{[15]}$. Compression of the LCFN can mimic a high lumbar disc herniation ${ }^{[16]}$. We treated 7 patients with a high lumbar herniated disc, and none had signs and symptoms of MP. It can get involved in the extraperitoneal approach to the anterior spine during handling of the psoas muscle. The nerve lies anteriorly and then traverses posterolateral to the psoas to reach the iliacus muscle. It can be injured at the anterior border of psoas. We approached the anterior border of psoas taking precaution to avoid damage the ascending lumbar vein, thus preventing MP. The harvesting of bone grafts from the iliac crest can damage this nerve and laparoscopic surgeons performing inguinal hernia repair must be aware of this problem ${ }^{[17,18]}$. The incidence of MP in our series was $12 \%$. There are few comparable series in the literature. However, Mirovsky in his series had 20\% incidence after spinal surgery in prone position. A much higher incidence in his series possibly relates to the HallRelton frame used by him instead of bolsters. Our bolsters are standard, 26 inches long and 13 inches in circumference. Smaller bolsters may avoid some of the vulnerable pressure points, as the surface area available is relatively smaller. The posts of the Hall-Relton frame over the anterior superior iliac crest should be adequately padded. As the LCFN of the thigh is sensory, it produces only discomfort without any muscle weakness. It is usually self-limiting and does not require any specific treatment. In the past, small doses of chlorpromazine produced good results in these patients. Surgical division or decompression of the LCFN is reserved for patients having persistent or severe pain. 


\section{Conclusion}

Although MP can be caused by conditions such as obesity, pregnancy, and sacroiliac dysfunction, it can also be a result of prolonged prone position after posterior spinal surgery. This is likely due to asymmetrical placement of the patient on the pelvic posts of various frames, resulting in bilateral compression of the LFCN. The severity of symptoms can help guide the extent of treatment; ranging from conservative measures, including loosening of tight clothing and administration of anti-in-flammatories, all the way to surgical neurectomy. Despite the numerous precautions that can be taken to prevent MP, it may always remain a risk during posterior spinal surgery due to the anatomic variation of the LFCN. Physicians should be mindful to keep the patient positioned symmetrically on the pelvic bolsters, while also making effort to reduce the length of surgery. Although MP can be caused by conditions such as obesity, pregnancy, and sacroiliac dysfunction, it can also be a result of prolonged prone position after posterior spinal surgery. This is likely due to asymmetrical placement of the patient on the pelvic posts of various frames, resulting in bilateral compression of the LFCN. The severity of symptoms can help guide the extent of treatment; ranging from conservative measures, including loosening of tight clothing and administration of anti-inflammatories, all the way to surgical neurectomy. Despite the numerous precautions that can be taken to prevent MP, it may always remain a risk during posterior spinal surgery due to the anatomic variation of the LFCN. Physicians should be mindful to keep the patient positioned symmetrically on the pelvic bolsters, while also making effort to reduce the length of surgery Although MP can be caused by sacroiliac dysfunction, it can also be the result of prolonged prone position after posterior spinal surgery. This is likely due to asymmetrical placement of the patient on the pelvic posts of various frames, resulting in bilateral compression of the LFCN. The severity of symptoms can help guide the treatment ranging from conservative measures like loosening of the tight clothing and administration of NSAIDS to all the way to surgical neurectomy. Despite the numerous precautions that can be taken to prevent MP, it may always remain a risk during posterior spinal surgeries due to the anatomic variation of the LFCN. Both surgeons and anaesthetist should be mindful to keep the patient positioned symmetrically on the pelvic bolsters, while also making efforts to reduce the length of the surgery. If the patient requires neurectomy to alleviate the symptoms, the localised anaesthesia will not be a hindrance to the quality of life.

Although MP can be caused by conditions such as obesity, pregnancy, and sacroiliac dysfunction, it can also be a result of prolonged prone position after posterior spinal surgery. This is likely due to asymmetrical placement of the patient on the pelvic posts of various frames, resulting in bilateral compression of the LFCN. The severity of symptoms can help guide the extent of treatment; ranging from conservative measures, including loosening of tight clothing and administration of anti-in-flammatories, all the way to surgical neurectomy. Despite the numerous precautions that can be taken to prevent MP, it may always remain a risk during posterior spinal surgery due to the anatomic variation of the LFCN. Physicians should be mindful to keep the patient positioned symmetrically on the pelvic bolsters, while also making effort to reduce the length of surgery Although MP can be caused by conditions such as obesity, pregnancy, and sacroiliac dysfunction, it can also be a result of prolonged prone position after posterior spinal surgery. This is likely due to asymmetrical placement of the patient on the pelvic posts of various frames, resulting in bilateral compression of the LFCN. The severity of symptoms can help guide the extent of treatment; ranging from conservative measures, including loosening of tight clothing and administration of anti-inflammatories, all the way to surgical neurectomy. Despite the numerous precautions that can be taken to prevent MP, it may always remain a risk during posterior spinal surgery due to the anatomic variation of the LFCN. Physicians should be mindful to keep the patient positioned symmetrically on the pelvic bolsters, while also making effort to reduce the length of surgery.

\section{References}

1. Pearce JMS. Meralgia paraesthetica (Bernhardt-Roth syndrome). Journal of Neurology, Neurosurgery \& Psychiatry. 2006; 77(1):84.

Doi:10.1136/jnnp.2005.072363. PMC 2117390. PMID 16 361600

2. Nahabedian MY, Dellon AL. Meralgia paresthetica: etiology, diagnosis, and outcome of surgical decompression. Ann Plast Surg. 1995; 35:590-4.

3. Holanda MM, Meira UM, Magalhaes FN, da Silva JA. [Surgical treatment of meralgia paresthetica: case report.] Arq Neuropsiquiatr. Portuguese, 2003; 61:288-90.

4. Grossman MG, Ducey SA, Nadler SS, Levy AS. Meralgia paresthetica: diagnosis and treatment. J Am Acad Orthop Sur g. 2001; 9:336-44.

5. Make your pencil jeans less dangerous to your health. Vanguard, 2012.

6. Mirovsky Y, Neuwirth M. Injuries to the lateral femoral cutaneous nerve during spine surgery. Spine. 2000; 25:1266-9.

7. Aszmann OC, Dellon ES, Dellon AL. Anatomical course of the lateral femoral cutaneous nerve and its susceptibility to compression and injury. Plast Reconstr Surg. 1997; 100:600-4.

8. Staal A. General discussion on pressure neuropathies. In: Vinken PS, Bruyn GW, editors. Handbook of Clinical Neurology- Diseases of the nerve. Amsterdam: North Holland Publishing Co. 1970; 7:276-84.

9. De Ridder VA, de Lange S, Popta JV. Anatomical variations of the lateral femoral cutaneous nerve and the consequences for surgery. J Orthop Trauma. 1999; 13:207-11.

10. Ivins GK. Meralgia paresthetica, the elusive diagnosis: clinical experience with 14 adult patients. Ann Surg. 2000; 232:281-6.

11. Geck MJ, Eismont FJ. Surgical options for the treatment of cervical spondylotic myelopathy. Orthop Clin North Am. 2002; 33:329-48.

12. Yamamoto $T$, Nagira $K$, Kurosak A. M. Meralgia paresthetica occurring 40 years after iliac bone graft harvesting: case report. Neurosurgery. 2001; 49:1455-7.

13. Simpson JA. Nerve injuries: General Aspects. In: Vinken PS, Bruyn GW, editors. Handbook of Clinical Neurology-Diseases of the nerve. Amsterdam: North Holland Publishing Co. 1970; 7:244-56.

14. Staal A. The Entrapment neuropathies. In: Vink en PS, Bruyn GW, editors. Handbook of Clinical Neurology Diseases of the nerve. Amsterdam: North Holland Publishing Co. 1970; 7:285-325.

15. Parsonnet V, Karasakalides A, Gielchinsky I, Hochberg M, Hussain SM. Meralgia paresthetica after coronary bypass surgery. J Thorac Cardiovasc Surg. 1991; 
101:219-21.

16. Trummer M, Flaschka G, Unger F, Eustacchio S. Lumbar disc herniation mimicking meralgia paresthetica: case report. Surg Neurol. 2000; 54:80-1.

17. Gilroy AM, Page DW. The clinical anatomy of laporoscopic inguinal hernia repair. Med J Singapore. 1996; 37:519-21.

18. Eubanks S, Newman L 3rd, Goehring L, Lucas GW, Adams CP, Mason E et al. Meralgia paresthetica: a complication of laparoscopic herniorrhaphy. Surg Laparosc Endosc. 1993; 3:381-5. 